\title{
HARMONIC ANALYSIS ON CERTAIN VECTOR SPACES
}

\author{
BY
}

\section{J. KUELBS( $\left({ }^{1}\right)$ AND V. MANDREKAR $\left({ }^{2}\right)$}

1. Introduction. Let $l$ denote the vector space of all sequences of real numbers with the topology of coordinate-wise convergence. For $0<p<\infty$ let $l_{p}$ denote the subset of $l$ consisting of all sequences which have $\|x\|=\left\{\sum_{i=1}^{\infty}\left|x_{i}\right|^{p}\right\}^{1 / p}$ finite where $x=\left(x_{1}, x_{2}, \ldots\right)$. Thus, for $1 \leqq p<\infty$, we have $\|\cdot\|$ as the usual norm for $l_{p}$, and for $0<p<1$ it is known that $\|\cdot\|^{p}$ generates a distance function under which $l_{p}$ is a complete linear metric space. Our main efforts in this paper are to generalize Bochner's theorem and Levy's continuity theorem to these $l_{p}$ spaces. For $p=2$ our work coincides with and simplifies some of the work of L. Gross in [3], [4], and [5], and is related to the work of V. Sazonov [8], [9]; Ju. V. Prohorov [7], [8]; and to that of N. N. Vakhania in [10], [11].

It should be mentioned that the case $p=2$ is brought into our work from two different points of view. One of these handles the situation for $0 \leqq p \leqq 2$ and the other for $2 \leqq p<\infty$. In both instances they agree with the work of L. Gross mentioned above. The tool that allows us to simplify Gross' work is a "stochastic inner product", defined in $\$ 3$, which allows the immediate application of usual measure theoretic manipulations. This idea of a stochastic inner product is suggested in the work of R. H. Cameron and R. E. Graves [1].

We will frequently think of the $l_{p}$ spaces as being subsets of $l$ and if $x \in l$ we define

$$
\begin{aligned}
P_{N} x & =\left(x_{1}, \ldots, x_{N}, 0,0, \ldots\right), \\
Q_{N} x & =\left(0, \ldots, 0, x_{N+1}, \ldots\right), \\
\mathscr{P}_{N} x & =\left(0, \ldots, 0, x_{N}, 0, \ldots\right) .
\end{aligned}
$$

This terminology is standard throughout the paper.

2. The Fourier transform (or characteristic functional) of a probability measure $\mu$ on the Borel subsets of a linear topological space $X$ is the function $\phi(x)$ on $X^{*}$ (the topological dual of $X$ ) such that

$$
\phi(x)=\int_{X} \exp \{i(x, y)\} d \mu(y)
$$

Received by the editors June 24,1968 .

( $\left.{ }^{1}\right)$ Supported in part by NSF Grant GP-7181.

( $\left.{ }^{2}\right)$ Sponsored by the Mathematics Research Center, United States Army, Madison, Wisconsin, under Contract No.: DA-31-124-ARO-D-462.

Copyright (C) 1970, American Mathematical Society 
The classical version of Bochner's theorem asserts that a function $\phi(x), x \in R_{n}$, is the Fourier transform of some probability measure on the Borel subsets of $R_{n}$ if and only if $\phi$ is positive definite, $\phi(0)=1$, and $\phi$ is continuous at $x=0$. In $l_{2}$, and hence in any real separable Hilbert space, it is possible to introduce a topology $\tau$ (which is determined by certain compact operators) such that a function $\phi$ on $l_{2}$ is the Fourier transform of some probability measure on the Borel subsets of $l_{2}$ if and only if $\phi$ is positive definite, $\phi(0)=1$, and $\phi$ is continuous at zero in the $\tau$ topology. Here we are, of course, identifying $l_{2}^{*}$ and $l_{2}$. The $l_{2}$ result is due independently to L. Gross [5] and to V. Sazonov's earlier work [9].

Before proving our analogue for Bochner's theorem for $l_{p}, 0<p \leqq 2$, we first take a closer look at the situation in $l_{2}$.

An operator on $l_{2}$ which is linear, symmetric, nonnegative, compact, and having finite trace will be called an $S$-operator. If $T$ is an $S$-operator on $l_{2}$ then it is well known that $T$ has the representation

$$
T x=\sum_{n=1}^{\infty} \lambda_{n}\left(x, e_{n}\right) e_{n}
$$

where $\left\{e_{n}\right\}$ is some orthonormal subset of $l_{2}, \lambda_{n} \geqq 0$, and $\sum_{n=1}^{\infty} \lambda_{n}<\infty$. The $S$-operator $T$ also has a representation as an infinite symmetric positive-definite matrix $T=\left\{t_{i k}\right\}$ where by positive-definite it is meant that $\sum_{i, k=1}^{n} t_{i k} x_{i} x_{k} \geqq 0$ for any integer $n$ and any $x \in R_{n}$. Furthermore, $t_{i k}=\left(T f_{i}, f_{k}\right)$ where $f_{j}$ is a sequence of all zeros and having 1 in the $j$ th position and hence $\sum_{i=1}^{\infty} t_{i i}=\sum_{j=1}^{\infty} \lambda_{j}<\infty$ where the $\lambda_{j}$ 's are as in (2.1). From the representation in $(2.1)$ it is easy to verify that $(T c x, c x)^{1 / 2}$ $=|c|(T x, x)^{1 / 2}$ for any real number $c$ and $(T(x+y),(x+y))^{1 / 2} \leqq(T x, x)^{1 / 2}+$ $(T y, y)^{1 / 2}$. Thus $(T x, x)^{1 / 2}$ is a seminorm on $l_{2}$. The $\tau$-topology on $l_{2}$ is the topology generated by taking as a subbase all translates of all sets of the form

$$
\left\{x \in l_{2}:(T x, x)<r\right\}
$$

where $r>0$ and $T$ is an $S$-operator.

As mentioned above, Gross [5] and Sazonov [9] have proved an analogue of Bochner's theorem which states that continuity in the $\tau$-topology at the origin is necessary and sufficient for a positive-definite complex-valued function $\phi$ on $l_{2}$ with $\phi(0)=1$ to be the Fourier transform of a positive finite measure. The following lemma demonstrates that $\tau$-continuity on $l_{2}$ is equivalent to ordinary continuity and continuity with respect to a certain directed set of distributions.

Lemma 2.1. Let $\phi(x)$ be a positive-definite functional defined on $l_{2}$ such that $\phi(0)=1$. Then $\phi(x)$ is $\tau$-continuous at zero (and hence everywhere on $l_{2}$ ) if and only if $\phi(x)$ is continuous in the norm topology and

$$
\phi(0)=\lim _{N \rightarrow \infty} \int_{l_{2}} \phi(x) \lambda\left(\sigma_{N}, d x\right)
$$


where $\lambda\left(\sigma_{N}, \cdot\right)$ denotes any Gaussian measure on $P_{N}\left(l_{2}\right)$ with density

$$
\left[(2 \pi)^{N} \prod_{j=1}^{N} \sigma_{N, j}^{2}\right]^{-1 / 2} \cdot \exp \left\{-\frac{1}{2} \sum_{j=1}^{N} \frac{x_{j}^{2}}{\sigma_{N, j}^{2}}\right\}
$$

such that $\max _{1 \leqq j \leqq N} \sigma_{N, j}^{2}$ tends to zero as $N$ tends to infinity.

Proof. Suppose $\phi$ is continuous on $l_{2}$ in the $\tau$-topology. Then we have for each $\varepsilon>0$ an $S$-operator such that $x \in E=\left\{x: \sum_{i, j=1}^{\infty} t_{i j} x_{i} x_{j}<1\right\}$ implies $|1-\phi(x)|<\varepsilon$. Thus if

we find

$$
A_{N}=\int_{l_{2}}[1-\phi(x)] \lambda\left(\sigma_{N}, d x\right)
$$

$$
\begin{aligned}
\left|A_{N}\right| & \leqq \varepsilon+2 \int_{E^{c}} \lambda\left(\sigma_{N}, d x\right) \\
& \leqq \varepsilon+2 \int_{l_{2}} \sum_{i, j=1}^{\infty} t_{i j} x_{i} x_{j} \lambda\left(\sigma_{N}, d x\right) \\
& \leqq \varepsilon+2 \sum_{j=1}^{N} t_{j j} \sigma_{N, j}^{2} \\
& \leqq \varepsilon+2 \max _{1 \leqq j \leqq N} \sigma_{N, j}^{2} \sum_{j=1}^{\infty} t_{j j} .
\end{aligned}
$$

Hence lim $\sup _{N}\left|A_{N}\right| \leqq \varepsilon$ so actually $A_{N}$ tends to zero, and $\tau$-continuity of $\phi$ implies (2.2) holds.

Since $\phi$ is positive definite and norm continuous it follows that $\phi\left(P_{k}(\cdot)\right)$ is a continuous, positive-definite function on $l$ for $k=1,2, \ldots$ Thus there exists a probability measure $\mu$ on the Borel subsets of $l$ whose finite dimensional distributions are determined by $\phi\left(P_{k}(\cdot)\right), k=1,2, \ldots$ Hence

$$
A_{N}=\int_{l}\left[1-\exp \left\{-\frac{1}{2} \sum_{j=1}^{N} \sigma_{N, j}^{2} x_{j}^{2}\right\}\right] d \mu(x) .
$$

Now $\lim _{N} A_{N}=0$ implies $\sum_{j=1}^{N} \sigma_{N, j}^{2} x_{i}^{2}$ tends to zero in $\mu$-measure as $\max _{1 \leqq j \leqq N} \sigma_{N j}^{2}$, tends to zero. This implies $\sum_{j=1}^{\infty} x_{j}^{2}$ is finite for almost all $x \in l$ with regard to the measure $\mu$ so $\mu\left(l_{2}\right)=1$. Hence if $\psi(x)=\int_{l_{2}} \exp \{i(x, y)\} d \mu(y)$ we find $\psi(x)=\phi(x)$ on $P_{k}\left(l_{2}\right)$ for $k=1,2, \ldots$ Since both functions are continuous on $l_{2}$ and $\bigcup_{k=1}^{\infty} P_{k} l_{2}$ is dense in $l_{2}$ this implies $\psi(x)=\phi(x)$ on $l_{2}$. Thus $\phi$ is the Fourier transform of $\mu$ and hence

$$
\phi(x)=\int_{l_{2}} \exp \{i(x, y)\} d \mu(y)
$$

Let $\varepsilon>0$ be given and choose a compact set $K \subseteq l_{2}$ such that $\mu\left(l_{2}-K\right)<\varepsilon / 2$. Then

$$
1-\operatorname{Re} \phi(x)=\int_{l_{2}}[1-\cos (x, y)] d \mu(y) \leqq \frac{1}{2} \int_{K}(x, y)^{2} d \mu(y)+\frac{\varepsilon}{2}
$$


and if we denote by $T$ the $S$-operator determined by the relation

$$
(T x, x)=\int_{K}(x, y)^{2} d \mu(y)
$$

it follows that $(T x, x)<\varepsilon$ implies $1-\operatorname{Re} \phi(x)<\varepsilon$. The $\tau$-continuity of $\phi$ at zero now follows since

$$
|1-\phi(x)|^{2} \leqq 2(1-\operatorname{Re} \phi(x)) .
$$

Thus continuity in the $\tau$-topology for positive-definite continuous functions on $l_{2}$ is equivalent to continuity with respect to a certain directed set of Gaussian distributions. This motivates our next result which generalizes Bochner's theorem to $l_{p}, 0<p \leqq 2$. In view of the previous lemma this agrees with the known results for $p=2$ in [5] and [9].

A function $\phi$ on $l_{p}^{*}$ will be called sequentially weak-star continuous if for each sequence $\left\{x_{n}\right\}$ in $l_{p}^{*}$ satisfying $\lim _{n}\left(y, x_{n}\right)=(y, x)$ for every $y \in l_{p}$ and some $x \in l_{p}^{*}$ we have $\lim _{n} \phi\left(x_{n}\right)=\phi(x)$.

TheOREM 2.1. Let $0<p \leqq 2$ and suppose $\phi(x)$ is a function defined on $l_{p}^{*}\left({ }^{3}\right)$. Then $\phi$ is the Fourier transform of a probability measure on $l_{p}$ if and only if $\phi$ is positive definite, $\phi(0)=1, \phi$ is sequentially weak-star continuous, and

$$
\phi(0)=\lim _{N \rightarrow \infty} \int_{l_{p}^{*}} \phi(x) \lambda_{p}\left(\varepsilon_{N}, d x\right) .
$$

Here $\lambda_{p}\left(\varepsilon_{N}, \cdot\right)$ denotes any stable distribution on $P_{N}\left(l_{p}^{*}\right)$ whose Fourier transform is

$$
\phi\left(t_{1}, \ldots, t_{N}\right)=\exp \left\{-\sum_{j=1}^{N} \varepsilon_{N, j}\left|t_{j}\right|^{p}\right\}
$$

where the $\varepsilon_{N, j}$ 's are positive and $\max _{1 \leqq j \leqq N} \varepsilon_{N, j}$ tends to zero as $N$ approaches infinity.

Proof. Let $B\left(\varepsilon_{N}\right)=\int_{l_{p}^{*}} \phi(x) \lambda_{p}\left(\varepsilon_{N}, d x\right)$. Since $\phi$ is positive definite, $\phi(0)=1$, and $\phi$ is continuous on $l_{p}^{*}$ there exists a probability measure $\mu$ on $l$ whose finite-dimensional distributions are determined by $\phi\left(P_{K}(\cdot)\right)$ for $K=1,2, \ldots$ Therefore,

$$
\begin{aligned}
B\left(\varepsilon_{N}\right) & =\int_{P_{N}\left(l_{p}^{*}\right)} \phi(x) \lambda_{p}\left(\varepsilon_{N}, d x\right) \\
& =\int_{P_{N}\left(l_{p}^{*}\right)} \int_{l} \exp \{i(x, y)\} d \mu(y) \lambda_{p}\left(\varepsilon_{N}, d x\right) \\
& =\int_{l} \int_{P_{N}\left(l_{p}^{*}\right)} \exp \{i(x, y)\} \lambda_{p}\left(\varepsilon_{N}, d x\right) d \mu(y) \\
& =\int_{l} \exp \left\{-\sum_{j=1}^{N} \varepsilon_{N, j}\left|y_{j}\right|^{p}\right\} d \mu(y) .
\end{aligned}
$$

$\left.{ }^{3}\right)$ If $0<p \leqq 1$ then $l_{p}^{*}=l_{\infty}$, the space of bounded sequences, with the usual sup norm. 
Since $B\left(\varepsilon_{N}\right)$ tends to $\phi(0)=1$ when $\max _{1 \leqq j \leqq N} \varepsilon_{N, j}$ tends to zero as $N$ approaches infinity we find $\max _{1 \leqq j \leqq N} \varepsilon_{N, j} \sum_{j=1}^{N}\left|y_{j}\right|^{p}$ tends to zero in $\mu$-measure. Thus $\sum_{j=1}^{\infty}\left|y_{j}\right|^{p}$ is finite with $\mu$-measure one. In other words, $\mu\left(l_{p}\right)=1$. If

$$
\psi(x)=\int_{l_{p}} \exp \{i(x, y)\} d \mu(y) \text { for } x \in l_{p}^{*}
$$

then $\psi(x)=\phi(x)$ on $\bigcup_{K=1}^{\infty} P_{K}\left(l_{p}^{*}\right)$ and since both $\psi$ and $\phi$ are continuous on $l_{p}^{*}$ it follows that $\phi$ actually equals $\psi$ on $l_{p}^{*}$, and hence is the Fourier transform of $\mu$.

On the other hand, if $\mu$ is a probability measure on $l_{p}$, then it is trivial to verify that

$$
\phi(x)=\int_{l_{p}} \exp \{i(x, y)\} d \mu(y) \quad\left(x \in l_{p}^{*}\right)
$$

is positive definite, continuous, and that $\phi(0)=1$. Further,

$$
\begin{aligned}
1 \geqq B\left(\varepsilon_{N}\right) & =\int_{l_{p}} \exp \left\{-\sum_{j=1}^{N} \varepsilon_{N, j}\left|y_{j}\right|^{p}\right\} d \mu(y) \\
& \geqq \int_{l_{p}} \exp \left\{-\max _{1 \leqq j \leqq N} \varepsilon_{N, j} \sum_{j=1}^{N}\left|y_{j}\right|^{p}\right\} d \mu(y)
\end{aligned}
$$

and the last integral converges to one so $B\left(\varepsilon_{N}\right)$ tends to $\phi(0)=1$ in the manner indicated.

In our investigation of probability measures on $l_{p}, 2 \leqq p<\infty$, via Fourier transforms, we found the following concept useful. A family of probability measures $\left\{\mu_{\alpha}: \alpha \in A\right\}$ on $l_{p}, 2 \leqq p<\infty$, is a $\lambda$-family for some $\lambda$ in the positive cone of $l_{p / 2}^{*}$ if for every $\varepsilon, \delta>0$ there is a sequence $\left\{\varepsilon_{N}\right\}$ such that

implies

$$
\mu_{\alpha}\left\{y \in l_{p}: \sum_{i=N+1}^{\infty} \lambda_{i} y_{i}^{2}<\delta\right\}>1-\varepsilon
$$

where $\lim _{N} \varepsilon_{N}=0$.

$$
\mu_{\alpha}\left\{y \in l_{p}: \sum_{i=N+1}^{\infty}\left|y_{i}\right|^{p}<\delta\right\}>1-\left(\varepsilon+\varepsilon_{N}\right)
$$

It is quite clear that any family of probability measures in $l_{2}$ is a $\lambda$-family for $\lambda=(1,1, \ldots)$.

We also need a generalization of the $\tau$-topology to $l_{q}(1 / q+1 / p=1)$. If $2 \leqq p<\infty$ then a linear operator $T$ from $l_{q}$ into $l_{p}$ is an $S_{p}$-operator if $T$ can be represented as an infinite symmetric positive-definite matrix $\left(t_{i j}\right)$ such that $\sum_{i=1}^{\infty}\left(t_{i i}\right)^{p / 2}$ is finite. Here, by positive-definite, we mean that $\sum_{i, j=1}^{n} t_{i j} x_{i} x_{j} \geqq 0$ for all $x \in R_{n}$ and all integers $n$. The $\tau_{p}$-topology $2 \leqq p<\infty$, is generated by taking as a subbase all translates of all sets of the form $\left\{x \in l_{p}^{*}:(T x, x)<r\right\}$ where $r>0$ and $T$ is an $S_{p}$ operator. Thus the $\tau_{2}$-topology is the $\tau$-topology.

The next theorem is a generalization of Prohorov's result [7] which handled the case $p=2$. 
THEOREM 2.2. A family $\left\{\mu_{\alpha}: \alpha \in A\right\}$ of probability measures on $l_{p}, 2 \leqq p<\infty$, is conditionally compact if and only if:

(a) For every $\mu_{\alpha}$ and $\varepsilon>0$ there exists an $S_{p}$-operator $T_{\alpha, \varepsilon}$ such that for $x \in l_{p}^{*}$

$$
\operatorname{Re}\left(1-\phi\left(x, \mu_{\alpha}\right)\right) \leqq\left(T_{\alpha, \varepsilon} x, x\right)+\varepsilon
$$

where $\phi\left(\cdot, \mu_{\alpha}\right)$ is the Fourier transform of $\mu_{\alpha}$.

(b) The norms of the operators $T_{\alpha, \varepsilon}$ are uniformly bounded by a constant $M_{\varepsilon}$.

(c) $\lim _{N} \sup _{\alpha} \sum_{k=N}^{\infty}\left(t_{k k}^{(\alpha, \varepsilon)}\right)^{p / 2}=0$.

(d) $\left\{\mu_{\alpha}: \alpha \in A\right\}$ is a $\lambda$-family for some $\lambda$ in the positive cone of $l_{p / 2}^{*}$.

The proof will depend on the following lemmas.

LEMMA 2.2. If $\left\{\mu_{\alpha}: \alpha \in A\right\}$ is a conditionally compact set of probability measures on $l_{p}, 2 \leqq p<\infty$, then $\left\{\mu_{\alpha}: \alpha \in A\right\}$ is a $\lambda$-family for any $\lambda$ in the positive cone of $l_{p / 2}^{*}$.

Proof. Let $\varepsilon, \delta$ be given and choose $\lambda \in l_{p / 2}^{*}$ where $\lambda_{k}>0$ for $k=1,2, \ldots$ Since $\left\{\mu_{\alpha}: \alpha \in A\right\}$ is conditionally compact there exists a compact set $K$ of $l_{p}$ such that $\mu_{\alpha}(K)>1-\varepsilon$ for each $\alpha \in A$. Thus there exists an $N$ such that for each $\alpha \in A$

$$
\mu_{\alpha}\left\{x \in l_{p}: \sum_{j=N+1}^{\infty}\left|x_{j}\right|^{p}<\delta\right\}>1-\varepsilon,
$$

so clearly $\left\{\mu_{\alpha}: \alpha \in A\right\}$ is a $\lambda$-family.

LEMMA 2.3. If $\left\{\mu_{\alpha}, \alpha \in A\right\}$ is conditionally compact then conditions (a), (b), (c), and (d) of Theorem 2.2 hold.

Proof. That (d) is true follows from Lemma 2.2. Let $\varepsilon>0$ be given. Since $\left\{\mu_{\alpha}: \alpha \in A\right\}$ is conditionally compact there exists a compact set $K$ such that $\mu_{\alpha}(K)>1-\varepsilon / 2$ for every $\alpha \in A$. Now

$$
\begin{aligned}
\operatorname{Re}\left(1-\phi\left(x, \mu_{\alpha}\right)\right) & =\operatorname{Re}\left[\int_{l_{p}}[1-\exp \{i(x, y)\}] d \mu_{\alpha}(y)\right] \\
& =\int_{l_{p}}[1-\cos (x, y)] d \mu_{\alpha}(y) \\
& \leqq \frac{1}{2} \int_{K}(x, y)^{2} d \mu_{\alpha}(y)+\varepsilon .
\end{aligned}
$$

Let $T_{\alpha, \varepsilon}=\left(t_{i j}^{(\alpha, \varepsilon)}\right)$ be the operator given by the matrix with $t_{i j}^{\alpha, \varepsilon}=\frac{1}{2} \int_{K} x_{i} x_{j} d \mu_{\alpha}(x)$. Clearly $T_{\alpha, \varepsilon}$ is positive definite and symmetric. Hence $T_{\alpha, \varepsilon}$ is an $S_{p}$-operator from $l_{p}^{*}$ into $l_{p}$ since

$$
\begin{aligned}
\sum_{i=1}^{\infty}\left(t_{i i}^{(\alpha, \varepsilon)}\right)^{p / 2} & =\sum_{i=1}^{\infty}\left(\frac{1}{2} \int_{K} x_{i}^{2} d \mu_{\alpha}(x)\right)^{p / 2} \leqq \sum_{i=1}^{\infty} \int_{K}\left|x_{i}\right|^{p} d \mu_{\alpha}(x) \\
& =\int_{K}\|x\|^{p} d \mu_{\alpha}(x) \leqq \sup _{x \in K}\|x\|^{p}
\end{aligned}
$$


and the last integral is finite because $K$ is a compact subset of $l_{p}$. Hence (a) holds. Since the norm of $T_{\alpha, \varepsilon}$ is dominated by

$$
\left[\sum_{i=1}^{\infty}\left(t_{i i}^{(\alpha, \varepsilon)}\right)^{p / 2}\right]^{2} \leqq\left[\sup _{x \in K}\|x\|^{p}\right]^{2}
$$

we have (b) and (c) following from (2.4).

LEMma 2.4. If $\left\{\mu_{\alpha}: \alpha \in A\right\}$ is a $\lambda$-family of probability measures on $l_{p}$ for some $\lambda$ in the positive cone of $l_{p / 2}^{*}$ then $\left\{\mu_{\alpha}: \alpha \in A\right\}$ is conditionally compact if:

(i) For every integer $N$ the Fourier transforms $\phi\left(P_{N} x, \mu_{\alpha}\right)$ are equicontinuous at zero in $P_{N} l_{p}^{*}$.

(ii) $\lim _{N} \sup _{\alpha} \lim _{k} J_{N, k}\left[1-\operatorname{Re} \phi\left(x, \mu_{\alpha}\right)\right]=0$ where

$$
J_{N, k}[\cdots]=\int_{\left(P_{N+k}-P_{N}\right) l_{p}^{*}}[\cdots] \lambda(N, k, d x)
$$

and $\lambda(N, k, \cdot)$ is the Gaussian product measure on $\left(P_{N+k}-P_{N}\right) l_{p}^{*}$ with each coordinate $x_{i}, N+1 \leqq i \leqq N+k$, being Gaussian with mean zero and variance $\lambda_{i}$.

Proof. Since $\phi\left(\cdot, \mu_{\alpha}\right)$ is the Fourier transform of $\mu_{\alpha}$ and $\lambda(N, k, \cdot)$ is symmetric about zero it follows that

$$
J_{N, k}\left[1-\operatorname{Re} \phi\left(x, \mu_{\alpha}\right)\right]=\int_{l_{p}}\left[1-\exp \left\{-\frac{1}{2} \sum_{i=N+1}^{N+k} \lambda_{i} x_{i}^{2}\right\}\right] d \mu_{\alpha}(x) .
$$

Thus (ii) implies that

$$
\lim _{N} \sup _{\alpha} \int_{l_{p}}\left[1-\exp \left\{-\frac{1}{2} \sum_{N+1}^{\infty} \lambda_{i} x_{i}^{2}\right\}\right] d \mu_{\alpha}(x)=0 .
$$

Let $0<\delta<1, \varepsilon>0$ be given and let $E_{N}=\left\{x \in l_{p}: \sum_{N+1}^{\infty} \lambda_{i} x_{i}^{2}<\delta / 2\right\}$. Since $t / 2 \leqq 1-e^{-t}$, $0 \leqq t \leqq 1$, it follows that

$$
\begin{aligned}
\mu_{\alpha}\left(E_{N}\right) & =1-\int_{E_{N}^{c}} d \mu_{\alpha} \geqq 1-\frac{8}{\delta} \int_{E_{N}^{c}}\left[1-\exp \left\{-\frac{1}{2} \sum_{i=N+1}^{\infty} \lambda_{i} x_{i}^{2}\right\}\right] d \mu_{\alpha}(x) \\
& \geqq 1-\frac{8}{\delta} \int_{l_{p}}\left[1-\exp \left\{-\frac{1}{2} \sum_{N+1}^{\infty} \lambda_{i} x_{i}^{2}\right\}\right] d \mu_{\alpha}(x) \geqq 1-\frac{\varepsilon}{3}
\end{aligned}
$$

for all $\alpha \in A$ and all $N$ sufficiently large due to (2.5). Since $\left\{\mu_{\alpha}: \alpha \in A\right\}$ is a $\lambda$-family we know there exists an $M$ such that for all $\alpha \in A$

$$
\mu_{\alpha}\left\{x \in l_{p}: \sum_{M+1}^{\infty}\left|x_{i}\right|^{p}<\frac{\delta}{2}\right\} \geqq 1-\frac{2 \varepsilon}{3} .
$$

By (i) we have $x_{1}, \ldots, x_{L} \in P_{M}\left(l_{p}\right)$ such that

$$
\mu_{\alpha}\left(P_{M}^{-1}\left(\bigcup_{k=1}^{L} S\left(x_{k}, \frac{\delta}{2}\right)\right)\right)>1-\frac{\varepsilon}{3}
$$


for all $\alpha \in A$ where $S(x, \gamma)=\left\{y \in l_{p}:\|x-y\|^{p}<\gamma\right\}$. Combining (2.6) with the above we see that for all $\alpha \in A$

$$
\mu_{\alpha}\left\{\bigcup_{k=1}^{L} S\left(x_{k}, \delta\right)\right\}>1-\varepsilon .
$$

Thus $\left\{\mu_{\alpha}: \alpha \in A\right\}$ is conditionally compact [7, p. 170] and the lemma is proved.

Proof of Theorem 2.2. If $\left\{\mu_{\alpha}: \alpha \in A\right\}$ is conditionally compact, Lemmas 2.2 and 2.3 demonstrate that (a), (b), (c), and (d) hold. Now if (a), (b), and (c) hold then (a) and (b) imply (i) of Lemma 2.4 and (a) and (c) imply (ii) since

$$
\begin{aligned}
\lim _{N} \sup _{\alpha} \lim _{k} J_{N, k}\left[\left(T_{\alpha, \varepsilon} x, x\right)+\varepsilon\right] \\
=\varepsilon+\lim _{N} \sup _{\alpha} \lim _{k} \int_{\left(P_{N+k}-P_{N}\right) l_{p}^{*}}\left(T_{\alpha, \varepsilon} x, x\right) \lambda(N, k, d x) \\
=\varepsilon+\lim _{N} \sup _{\alpha} \lim _{k} \sum_{i, j=N+1}^{N+k} \int_{\left(P_{N+k}-P_{N}\right) l_{p}^{*}} t_{i j}^{(\alpha, \varepsilon)} x_{i} x_{j} \lambda(N, k, d x) \\
=\varepsilon+\lim _{N} \sup _{\alpha} \sum_{i=N+1}^{\infty} \lambda_{i} t_{i i}^{(\alpha, \varepsilon)} .
\end{aligned}
$$

That is, since $\lambda=\left(\lambda_{1}, \lambda_{2}, \ldots\right)$ is an element in the positive cone of $l_{p / 2}^{*}$ we have, by (c), that

$$
\lim _{N} \sup _{\alpha} \sum_{i=N+1}^{\infty} \lambda_{i} t_{i i}^{(\alpha, \varepsilon)}
$$

and hence (ii) holds. Finally, (i), (ii), and (d) imply that $\left\{\mu_{\alpha}: \alpha \in A\right\}$ is conditionally compact so Theorem 2.2 is proved.

We now turn to Bochner's theorem on $l_{p}, 2 \leqq p<\infty$.

THEOREM 2.3. A function $\phi(x)$ defined on $l_{p}^{*}$ is the Fourier transform of a probability measure on the Borel subsets of $l_{p}, 2 \leqq p<\infty$, if and only if

(i) $\phi(0)=1$ and $\phi$ is positive definite on $l_{p}^{*}$.

(ii) $\phi$ is continuous at zero in the $\tau_{p}$-topology on $l_{p}^{*}$.

(iii) The family of measures $\left\{\mu_{n}\right\}$ corresponding to $\phi\left(P_{n}(\cdot)\right)$ is a $\lambda$-family for some $\lambda$ in the positive cone of $l_{p / 2}^{*}$.

Proof. If $\mu$ is a probability measure on $l_{p}$ with Fourier transform $\phi$ then $\phi$ clearly satisfies (i). Let $\varepsilon>0$ be given and choose a compact set $K$ of $l_{p}$ such that $\mu(K)$ $>1-\varepsilon / 2$. Then for $x \in l_{p}^{*}$

$$
\begin{aligned}
\int_{l_{p}}[1-\cos (x, y)] d \mu(y) & \leqq \frac{1}{2} \int_{K}(x, y)^{2} d \mu(y)+\varepsilon \\
& \leqq \frac{1}{2} \sum_{i, j=1}^{\infty} x_{i} x_{j} \int_{K} y_{i} y_{j} d \mu(y)+\varepsilon
\end{aligned}
$$

Now let $T=\left(t_{i j}\right)$ where $t_{i j}=\int_{K} y_{i} y_{j} d \mu(y)$. Then

$$
\sum_{i=1}^{\infty}\left(t_{i i}\right)^{p / 2}=\sum_{i=1}^{\infty}\left(\int_{K} y_{i}^{2} d \mu(y)\right)^{p / 2} \leqq \sum_{i=1}^{\infty} \int_{K}\left|y_{i}\right|^{p} d \mu(y)=\int_{K}\|y\|^{p} d \mu(y)<\infty
$$


and this implies, using (2.7),

$$
|1-\phi(x)|^{2} \leqq 2[1-\operatorname{Re} \phi(x)] \leqq(T x, x)+\varepsilon
$$

for any $x \in l_{p}^{*}$. Thus $\phi$ is $\tau_{p}$-continuous at zero and (ii) holds. We now need only show that $\left\{\mu_{n}\right\}$ satisfies (iii). Let $f$ be any bounded continuous function on $l_{p}$ and notice that $\mu_{n}(A)=\mu\left(P_{n}^{-1}(A)\right)$ for every Borel set $A$. Thus

$$
\lim _{n} \int_{l_{p}} f d \mu_{n}=\lim _{n} \int_{l_{p}} f\left(P_{n}(x)\right) d \mu(x)=\int_{l_{p}} f d \mu
$$

and hence $\left\{\mu_{n}\right\}$ converges weakly to $\mu$. By Lemma $2.2\left\{\mu_{n}\right\}$ satisfies (iii).

If (i), (ii), and (iii) hold we show that Theorem 2.2 is applicable to the sequence $\left\{\mu_{n}\right\}$. Let $\varepsilon>0$ be given. Using (ii) there exists an $S_{p}$-operator $T^{\varepsilon}$ on $l_{p}^{*}$ such that

$$
1-\operatorname{Re} \phi(x) \leqq\left(T^{\varepsilon} x, x\right)+\varepsilon .
$$

Since $\phi\left(x, \mu_{n}\right)=\phi\left(P_{n} x\right)$ we see that

$$
1-\operatorname{Re} \phi\left(x, \mu_{n}\right) \leqq\left(T^{\varepsilon} P_{n} x, P_{n} x\right)+\varepsilon=\left(T_{n}^{\varepsilon} x, x\right)+\varepsilon
$$

for $n=1,2, \ldots$, where $T_{n}^{\varepsilon}=P_{n} T^{\varepsilon} P_{n}$. Thus (a), (b), (c) of Theorem 2.2 hold and (iii) is equivalent to (d) so $\left\{\mu_{n}\right\}$ is a conditionally compact sequence of probability measures on $l_{p}$. Hence if $\left\{\mu_{n_{k}}\right\}$ is a convergent subsequence of $\left\{\mu_{n}\right\}$ converging to the measure $\mu$ on $l_{p}$ then $\phi\left(x, \mu_{n_{k}}\right)=\phi\left(P_{n_{k}}(x)\right)$ so $\lim _{k} \phi\left(x, \mu_{n_{k}}\right)=\phi(x)$. This implies the Fourier transform of $\mu$ is $\phi(x)$ and the theorem is proved.

We now consider some easy examples which indicate the independence of the conditions (i), (ii), and (iii) in Theorem 2.3. The first example shows that (i) and (iii) do not imply (ii). Let $2 \leqq p<\infty$ and assume $\psi(x), x \in l_{p}^{*}$, is the Fourier transform of a probability measure $\mu$ on $l_{p}$. Let $\phi(x)=\psi(x)$ if $x \in \bigcup_{k=1}^{\infty} P_{k}\left(l_{p}^{*}\right)$ and be zero elsewhere. Then $\phi$ satisfies (i) and since $\phi\left(P_{n}(\cdot)\right)=\psi\left(P_{n}(\cdot)\right)=\psi\left(\cdot, \mu_{n}\right)$ and $\left\{\mu_{n}\right\}$ converges weakly to $\mu$ it follows that $\left\{\mu_{n}\right\}$ is a $\lambda$-family. Furthermore, $\phi$ is not continuous at zero in the norm topology of $l_{p}^{*}$ and hence not in the $\tau_{p}$-topology so (ii) does not hold. The second example shows that (i) and (ii) do not imply (iii). Take $p=4$ and consider the probability measure $\mu$ on $l$ such that the coordinates are independent functions each with distribution

$$
\mu\left\{y \in l: y_{k}= \pm 1\right\}=1 / k, \quad \mu\left\{y \in l: y_{k}=0\right\}=1-2 / k .
$$

Now $l_{4}^{*}=l_{4 / 3}$ so for $x \in l_{4 / 3}$ we have

$$
\begin{aligned}
\phi(x)=\int_{l} \exp \{i(x, y)\} d \mu(y) & =\prod_{k=1}^{\infty}\left[\int_{l} \exp \left\{i x_{k} y_{k}\right\} d \mu(y)\right]^{\left({ }^{4}\right)} \\
& =\prod_{k=1}^{\infty}\left[1-\frac{2}{k}\left(1-\cos x_{k}\right)\right]
\end{aligned}
$$

$\left({ }^{4}\right)$ This integration will be justified in the next section after Lemma 3.1 . 
and since $0 \leqq 1-\cos t \leqq t^{2} / 2$ we have

$$
\sum_{k=1}^{\infty} \frac{\left[1-\cos x_{k}\right]}{k} \leqq \sum_{k=1}^{\infty} \frac{x_{k}^{2}}{k}<\infty
$$

because $x \in l_{4 / 3} \subset l_{2}$. Thus $\phi$ satisfies (i) and is continuous in the norm topology on $l_{4 / 3}$. For $x \in P_{N} l_{4 / 3}$ we have

$$
|\phi(x)-1|^{2} \leqq 2 \int_{l}(1-\cos (x, y)) d \mu(y) \leqq \int_{l}(x, y)^{2} d \mu(y)=\sum_{i=1}^{N} \frac{x_{i}^{2}}{i} \leqq \sum_{i=1}^{\infty} \frac{x_{i}^{2}}{i}
$$

and the last term is finite for $x \in l_{4 / 3} \subset l_{2}$. Let $T=\left(t_{i j}\right)$ where $t_{i i}=1 / i$ and $t_{i j}=0$ for $i \neq j$. Then $(T x, x)=\sum_{i=1}^{\infty} x_{i}^{2} / i$ for $x \in l_{4 / 3}$ and $\sum_{i=1}^{\infty}(1 / i)^{p / 2}<\infty$, so $T$ is an $S_{p-}$ operator such that for $x \in P_{N} l_{4 / 3}$

$$
|\phi(x)-1|^{2} \leqq(T x, x) \quad(N=1,2, \ldots) .
$$

Now both sides are norm continuous functions on $l_{4 / 3}$ and since $\bigcup_{N=1}^{\infty} P_{N} l_{4 / 3}$ is dense in $l_{4 / 3}$ we have $|\phi(x)-1| \leqq(T x, x)$ for all $x \in l_{4 / 3}$. Thus $\phi$ satisfies (ii) of Theorem 2.3. Using the Borel-Cantelli lemma it is clear that

$$
\mu\left\{y \in l: \sum_{k=1}^{\infty}\left|y_{k}\right|^{p}<\infty\right\}=0
$$

for all $p \geqq 2$ and in particular for $p=4$ so (iii) cannot hold.

This last example contradicts a conjecture of Ju. V. Prohorov and V. Sazonov. In view of the results of [10] and [11] they seem to imply in [8] that continuity in the $S_{p}$-topology would be sufficient for a positive definite function $\phi$, with $\phi(0)=1$, to be the Fourier transform of a probability measure on $l_{p}$. Our example shows that this is not enough.

3. For each $p, 0<p \leqq 2$, we denote by $\lambda_{p}$ the probability measure on the Borel subsets $\mathscr{B}$ of $l$ formed by taking the product measure on $l$ such that the coordinate functions have independent symmetric stable laws with Fourier transform $\exp \left\{-\frac{1}{2}|t|^{p}\right\}$.

Lemma 3.1. If $\mu$ is a probability measure on the Borel sets $\mathscr{C}$ of $l_{p}, 0<p \leqq 2$, then the function

$$
(x, y)=\lim _{N} \sum_{k=1}^{N} x_{k} y_{k}
$$

is a $\mathscr{B} \times \mathscr{C}$-measurable function on $l \times l_{p}$ where $x=\left(x_{1}, x_{2}, \ldots\right), y=\left(y_{1}, y_{2}, \ldots\right)$, and $\left(\lambda_{p} \times \mu\right)\{|(x, y)|<\infty\}=1$.

Proof. Let $F(x, y)=(x, y)$ if $(x, y)$ exists and is finite, and be infinity otherwise. Then $F(x, y)$ is $\mathscr{B} \times \mathscr{C}$-measurable (it is the limit of $\mathscr{B} \times \mathscr{C}$ measurable functions), and if $E=\{|F(x, y)|<\infty\}$ then we claim $\left(\lambda_{p} \times \mu\right)(E)=1$. To see this notice that for each $y \in l_{p}$ we have $Z_{1}(x)=x_{1} y_{1}, \ldots, Z_{k}(x)=x_{k} y_{k}, \ldots$ a sequence of independent 
stable random variables such that

$$
\int_{l} \exp \left\{i t Z_{k}(x)\right\} d \lambda_{p}(x)=\exp \left\{-\frac{1}{2}\left|y_{k} t\right|^{p}\right\}
$$

Hence $\sum_{k=1}^{\infty} Z_{k}$ converges in distribution, and hence almost everywhere, to a stable random variable with Fourier transform

$$
\exp \left\{-\frac{1}{2} \sum_{k=1}^{\infty}\left|y_{k}\right|^{p}|t|^{p}\right\} .
$$

Thus for fixed $y \in l_{p}, F(x, y)$ is finite for almost all $x \in l$. Since $F$ is jointly measurable the set $E$ is jointly measurable and

$$
\left(\lambda_{p} \times \mu\right)(E)=\int_{l_{p}}\left[\int_{l} I_{E}(x, y) d \lambda_{p}(x)\right] d \mu(y)=1
$$

since the inner integral is one for all $y \in l_{p}$.

Henceforth we will use $(x, y)$ in both the usual sense when $x, y \in l_{2}$ or $x \in l_{p}$ and $y \in l_{q}$, or in a "stochastic sense" such as defined in Lemma 3.1. The task of deciding which way the inner product is being used is trivial and hence will not always be mentioned. For example, the stocastic inner product used in (2.8) can be rigorously obtained by repeating the proof of Lemma 3.1, and the second equality is then only an application of the bounded convergence theorem.

If $\mu$ is a measure on the Borel sets of $l_{p}, 0<p \leqq 2$, and

$$
\tilde{\phi}(x)=\int_{l_{p}} \exp \{i(x, y)\} d \mu(y) \quad(x \in l)
$$

then $\bar{\phi}$ is a Borel measurable function on $l$ which is finite almost everywhere with respect to the measure $\lambda_{p}$ and which is equal to

$$
\phi(x)=\int_{l_{p}} \exp \{i(x, y)\} d \mu(y)
$$

for all $x \in l_{p}^{*}$. Thus $\phi$ is an extension of $\phi$ from $l_{p}^{*}$ to $l$. When dealing with $\phi$ we will always choose a version which agrees with $\phi$ on $l_{p}^{*}$ and we will refer to $\phi$ as an extended Fourier transform.

We now state the continuity theorem for $l_{p}, 0<p \leqq 2$.

THEOREM 3.1. If $0<p \leqq 2$ and $\left\{\mu_{k}\right\}$ is a sequence of probability measures on $l_{p}$ with Fourier transforms $\left\{\phi_{k}\right\}$ defined on $l_{p}^{*}$, then $\left\{\mu_{k}\right\}$ converges weakly to a measure $\mu$ with Fourier transform $\phi$ if and only if $\phi_{k}$ converges in probability to $\phi$ with respect to the measure $\lambda_{p}$ and $\left\{\phi_{k}\right\}$ converges to $\phi$ on $l_{p}^{*}$.

Actually, Theorem 3.1 is not quite equivalent to L. Gross' result (the case $p=2$ ), and in $\S 4$ we will obtain the equivalent result for $p=2$. We wish to point out that our proof will depend only on the stochastic inner product we have defined, and 
the great smoothness of $\tau$-continuous functions (see Lemma 4.2). This contrasts very much with the highly analytical proof given by Gross. We first proceed with several lemmas and the proof of Theorem 3.1. The first lemma uses an idea from J. Feldman's [2].

LEMMA 3.2. If $\left\{\mu_{\alpha}: \alpha \in A\right\}$ is a family of probability measures on $l_{p}, 0<p \leqq 2$ such that

$$
\lim _{N \rightarrow \infty: \gamma \downarrow 0} \sup _{\alpha} J_{N, \gamma}\left(\mu_{\alpha}\right)=0
$$

where

$$
J_{N, \gamma}\left(\mu_{\alpha}\right)=\int_{l_{p}}\left[1-\exp \left\{-\frac{1}{2}\left\|\gamma P_{N} x+Q_{N} x\right\|^{p}\right\}\right] d \mu_{\alpha}(x)
$$

then $\left\{\mu_{\alpha}: \alpha \in A\right\}$ is conditionally compact.

Proof. Let $\varepsilon>0,0<\delta \leqq 1$ be given and define

$$
E=\left\{x \in l_{p}:\left\|\gamma P_{N} x+Q_{N} x\right\| \geqq \delta / 2\right\} .
$$

If $0 \leqq t \leqq 1$ then $t / 2 \leqq 1-e^{-t}$ so we find

$$
\mu_{\alpha}(E) \leqq 4\left(\frac{2}{\delta}\right)^{p} \int_{l_{p}}\left[1-\exp \left\{-\frac{1}{2}\left\|\gamma P_{N} x+Q_{N} x\right\|^{p}\right\}\right] d \mu_{\alpha}(x)=\frac{2^{p+2}}{\delta^{p}} J_{N, \gamma}\left(\mu_{\alpha}\right) .
$$

By our condition there exists $N_{0}(\varepsilon, \delta)$ and $\gamma_{0}(\varepsilon, \delta)$ such that $N \geqq N_{0}, \gamma \leqq \gamma_{0}$ implies

$$
J_{N, \gamma}\left(\mu_{\alpha}\right) \leqq \varepsilon\left(\delta^{p} / 2^{p+2}\right) \quad(\alpha \in A)
$$

Thus

$$
\mu_{\alpha}\left\{x \in l_{p}:\left\|\gamma P_{N} x+Q_{N} x\right\|<\delta / 2\right\} \geqq 1-\varepsilon
$$

for $\gamma \leqq \gamma_{0}, N \geqq N_{0}$, and all $\mu_{\alpha}$. Further, $x \in E^{C}$ implies $\left\|P_{N} x\right\|<(\delta / 2 \gamma)$, so we let $x_{1}, \ldots, x_{r}$ be in $P_{N} l_{p}$ such that $\left\|x_{j}\right\|<\delta / 2 \gamma$ and such that for all $x \in l_{p}$ with $\left\|P_{N} x\right\|$ $<\delta / 2 \gamma$ we have

$$
\min _{1 \leqq j \leqq r}\left\|P_{N} x-x_{j}\right\|<\delta / 2 .
$$

Then $\mu_{\alpha}\left\{\bigcup_{j=1}^{r} S\left(x_{j}, \delta\right)\right\} \geqq 1-\varepsilon$ for all $\mu_{\alpha}$ where

$$
S(x, \delta)=\left\{y \in l_{p}:\|y-x\|<\delta\right\} .
$$

That is,

$$
S\left(x_{j}, \delta\right)=\left\{y \in l_{p}:\left\|y-x_{j}\right\|<\delta\right\} \supseteq\left\{y \in l_{p}:\left\|P_{N} y-x_{j}\right\|<\delta / 2,\left\|Q_{N} y\right\|<\delta / 2\right\}
$$

and hence $E^{c} \subseteq \bigcup_{j=1}^{r} S\left(x_{j}, \delta\right)$. Thus $\left\{\mu_{\alpha}: \alpha \in A\right\}$ is conditionally compact.

LEMMA 3.3. If $\left\{\mu_{k}\right\}$ is a sequence of probability measures on $l_{p}$ such that $\left\{\phi_{k}\right\}$ converges in $\lambda_{p}$-measure to $\phi$ where $\phi$ is the Fourier transform of a measure $\mu$ in $l_{p}$, then

$$
\lim _{N \rightarrow \infty ; \gamma \downarrow 0} \sup _{k} J_{N, \gamma}\left(\mu_{k}\right)=0 .
$$


Proof. First of all observe that

$$
\begin{aligned}
J_{N, \gamma}\left(\mu_{k}\right) & =\int_{l_{p}}\left[1-\exp \left\{-\frac{1}{2}\left\|\gamma P_{N} y+Q_{N} y\right\|^{p}\right\}\right] d \mu_{k}(y) \\
& =\int_{l}\left[1-\bar{\phi}_{k}\left(\gamma P_{N} x+Q_{N} x\right)\right] d \lambda_{p}(x)
\end{aligned}
$$

Thus

$$
\lim _{k \rightarrow \infty} J_{N, \gamma}\left(\mu_{k}\right)=\int_{l}\left[1-\tilde{\phi}\left(\gamma P_{N} x+Q_{N} x\right)\right] d \lambda_{p}(x)
$$

since $\left\{\tilde{\phi}_{k}\right\}$ converging in $\lambda_{p}$-measure to $\tilde{\phi}$ implies $\left\{\tilde{\phi}_{k} \circ T_{N, \gamma}\right\}$ converges in measure to $\tilde{\phi} \circ T_{N, \gamma}$ where $T_{N, \gamma}(x)=\gamma P_{N} x+Q_{N} x$. Hence

$$
\begin{aligned}
\lim _{N \rightarrow \infty ; \gamma \downarrow 0} \sup _{k} J_{N, \gamma}\left(\mu_{k}\right) & =\lim _{N \rightarrow \infty: \gamma \downarrow 0} \int_{l}\left[1-\tilde{\phi}\left(\gamma P_{N} x+Q_{N} x\right)\right] d \lambda_{p}(x) \\
& =\lim _{N \rightarrow \infty ; \gamma \downarrow 0} \int_{l} \int_{l_{p}}\left[1-\exp \left\{i\left(\gamma P_{N} x+Q_{N} x, y\right)\right\}\right] d \mu(y) d \lambda_{p}(x) \\
& =\lim _{N \rightarrow \infty ; \gamma \downarrow 0} \int_{l_{p}}\left[1-\exp \left\{-\frac{1}{2}\left\|\gamma P_{N} y+Q_{N} y\right\|^{p}\right\}\right] d \mu(y)=0
\end{aligned}
$$

as was to be shown.

Proof of Theorem 3.1. We first assume $\left\{\mu_{k}\right\}$ converges weakly to $\mu$ on $l_{p}$. Then $\lim _{k} \phi_{k}(x)=\phi(x)$ on $l_{p}^{*}$ and

$$
\int_{l}\left|\tilde{\phi}_{k}-\tilde{\phi}\right|^{2} d \lambda_{p}=\int_{l}\left|\tilde{\phi}_{k}\right|^{2} d \lambda_{p}-\int_{l} \tilde{\phi}_{k} \tilde{\psi} d \lambda_{p}-\int_{l} \tilde{\psi}_{k} \tilde{\phi} d \lambda_{p}+\int_{l}|\tilde{\phi}|^{2} d \lambda_{p}
$$

where $\tilde{\psi}, \tilde{\psi}_{k}$ are the conjugates of $\tilde{\phi}$ and $\tilde{\phi}_{k}$. Furthermore, $\left\{\mu_{k}\right\}$ converging weakly to $\mu$ implies $\left\{\mu_{k} \times \mu_{k}\right\}$ converges weakly to $\mu \times \mu$ and hence

$$
\begin{aligned}
\lim _{k} \int_{l}\left|\tilde{\phi}_{k}\right|^{2} d \lambda_{p} & =\lim _{k} \int_{l} \int_{l_{p}} e^{i(x, y)} d \mu_{k}(y) \cdot \int_{l_{p}} e^{-i(x, z)} d \mu_{k}(z) d \lambda_{p}(x) \\
& =\lim _{k} \int_{l_{p}} \int_{l_{p}} \int_{l} e^{i(x, y-z)} d \lambda_{p}(x) d \mu_{k}(y) d \mu_{k}(z) \\
& =\lim _{k} \int_{l_{p}} \int_{l_{p}} \exp \left(-\frac{1}{2}\|y-z\|^{p}\right) d \mu_{k}(y) d \mu_{k}(z) \\
& =\int_{l_{p}} \int_{l_{p}} \exp \left(-\frac{1}{2}\|y-z\|^{p}\right) d \mu(y) d \mu(z) \\
& =A .
\end{aligned}
$$

Similarly, $\int_{l} \phi_{k} \tilde{\psi} d \lambda_{p}$ and $\int_{l} \tilde{\psi}_{k} \tilde{\phi} d \lambda_{p}$ both converge to $A$ as $k$ approaches infinity. Thus we have $\left\{\tilde{\phi}_{k}\right\}$ converging in mean-square to $\tilde{\phi}$, and hence $\left\{\tilde{\phi}_{k}\right\}$ converges to $\phi$ in $\lambda_{p}$-measure.

On the other hand, if $\left\{\tilde{\phi}_{k}\right\}$ converges to $\tilde{\phi}$ in $\lambda_{p}$-measure on $l$ where $\phi$ is the Fourier transform of a probability measure on $l_{p}$ we know, by applying Lemmas 3.2 and 3.3, that $\left\{\mu_{k}\right\}$ is conditionally compact. Hence there is a subsequence $\left\{\mu_{k j}\right\}$ converging weakly to a probability measure $\nu$ with Fourier transform $\psi$. Then $\psi=\lim _{j} \phi_{k_{j}}=\phi$ 
and hence $\left\{\mu_{k_{j}}\right\}$ converges weakly to $\mu$ by the uniqueness of the Fourier transforms for measure on $l_{p}$. Furthermore, this shows that any convergent subsequence of $\left\{\mu_{k}\right\}$ must converge to $\mu$ and hence $\left\{\mu_{k}\right\}$ actually converges to $\mu$ because every subsequence has a convergent subsequence going weakly to $\mu$.

4. We now turn to the special case $p=2$ and prove the continuity theorem given by L. Gross in [5]. As mentioned previously, our proof mainly depends on the stochastic inner product and the smoothness of $\tau$-continuous functions on $l_{2}$. This is in contrast to the analytical approach of L. Gross [5] and J. Feldman [2]. The Gaussian measure $\lambda_{2}$ on $l$ will be denoted by $P(\cdot)$ throughout this section.

Lemma 4.1. Let $T$ be an $S$-operator on $l_{2}$. Then $T(x)=\sum_{i} \gamma_{i}\left(x, \alpha_{i}\right) \alpha_{i}$ where $\gamma_{i}>0$, $\sum_{i} \gamma_{i}<\infty,\left\{\alpha_{1}, \alpha_{2}, \cdots\right\}$ is an orthonormal family, and

$$
(T x, x)=\sum_{i} \gamma_{i}\left(x, \alpha_{i}\right)^{2}
$$

is finite on a linear subset of l of P-measure one. Furthermore, if

$$
Z_{N}(x)=\left(T Q_{N} x, Q_{N} x\right)
$$

then $\lim _{N} Z_{N}(x)=0$ on some linear subset $\mathscr{E}$ of l such that $P(\mathscr{E})=1$.

Proof. Since $T$ is an $S$-operator the given representation for $T$ is well known. That $(T x, x)$ is finite on a subset of measure one follows since $\left\{\left(x, \alpha_{i}\right)\right\}$ is a sequence of independent Gaussian functionals with mean zero and variance one, and since $\sum_{i} \gamma_{i}<\infty$. The linearity of this subset follows since

$$
(T(x+y), x+y) \leqq\left\{(T x, x)^{1 / 2}+(T y, y)^{1 / 2}\right\}^{2} .
$$

The linearity of the subset of $l$ where $\lim _{N} Z_{N}(x)=0$ follows in the same way. Hence all that remains is to show that $\lim _{N} Z_{N}(x)=0$ for almost all $x \in l$. First we observe that

$$
E\left(Z_{N}(x)\right)=\sum_{i} \gamma_{i} E\left(Q_{N} x, \alpha_{i}\right)^{2}=\sum_{i} \gamma_{i}\left(Q_{N} \alpha_{i}, \alpha_{i}\right)
$$

so $\lim _{N} E\left(Z_{N}(x)\right)=0$.

Furthermore, $\left\{Z_{N}\right)_{N}$ is a reversed submartingale so the convergence to zero almost everywhere $(P)$ follows. That is, for all $A \in \mathscr{B}\left(Z_{N+1}, Z_{N+2}, \ldots\right)$

$$
\begin{aligned}
\int_{A} E\left(Z_{N} \mid Z_{N+1}, Z_{N+2}, \ldots\right) d P & =\int_{A} Z_{N} d P=\int_{A} \sum_{i} \gamma_{i}\left(Q_{N} x, \alpha_{i}\right)^{2} d P \\
& =\sum_{i} \int_{A} \gamma_{i}\left[\left(\mathscr{P}_{N+1} x, \alpha_{i}\right)+\left(Q_{N+1} x, \alpha_{i}\right)\right]^{2} d P \\
& =\sum_{i} \gamma_{i} \int_{A}\left\{\left(\mathscr{P}_{N+1} x, \alpha_{i}\right)^{2}+\left(Q_{N+1} x, \alpha_{i}\right)\right\}^{2} d P \\
& \geqq \sum_{i} \gamma_{i} \int_{A}\left(Q_{N+1} x, \alpha_{i}\right)^{2} d P \\
& =\int_{A} Z_{N+1} d P
\end{aligned}
$$


where the equality preceding the inequality follows since $\left(\mathscr{P}_{N+1} x, \alpha_{i}\right)$ and $\left(Q_{N+1} x, \alpha_{i}\right)$ are independent with mean zero and $A \in \mathscr{B}\left(Z_{N+1}, Z_{N+2}, \ldots\right)$. Thus

$$
E\left(Z_{N} \mid Z_{N+1}, Z_{N+2}, \ldots\right) \geqq Z_{N+1}
$$

so $\left\{Z_{N}\right\}_{N}$ is a reversed submartingale [6, p. 393].

LeMmA 4.2. If $\phi(x)$ is uniformly $\tau$-continuous on $l_{2}$ then $\phi(x)$ can be uniquely extended, call the extension $\tilde{\phi}$, to be uniformly continuous on a linear subset $\mathscr{E}$ of $l$ such that

(i) $P(\mathscr{E})=1$.

(ii) For $x \in \mathscr{E}$ we have $\tilde{\phi}(x)=\lim _{N} \phi\left(P_{N} x\right)$.

(iii) $\lim _{N \rightarrow \infty ; \gamma \rightarrow 0} \delta\left(\gamma P_{N} x+Q_{N} x\right)=\tilde{\phi}(0)=\phi(0)$ for $x \in \mathscr{E}$.

Proof. Since $\phi(x)$ is uniformly $\tau$-continuous there exists, for each integer $k$, an $S$-operator $T_{k}$ such that if $E_{k}=\left\{x \in l_{2}:\left(T_{k} x, x\right)<1\right\}$ then

$$
|\phi(y+x)-\phi(y)|<1 / k \quad\left(x \in E_{k}, y \in l_{2}\right) .
$$

Choose at least one $T_{k}$ such that $\left(T_{k} x, x\right)>0$ unless $x=0$. Let

$$
\begin{array}{r}
\mathscr{E}_{k}=\left\{x \in l:\left(T_{k} Q_{N} x, Q_{N} x\right)<\infty \text { for } N=0,1,2, \ldots ; \lim _{N}\left(T_{k} Q_{N} x, Q_{N} x\right)=0\right\} \\
\text { for } k=1,2, \ldots
\end{array}
$$

Then $P\left(\mathscr{E}_{k}\right)=1$ and $\mathscr{E}_{k}$ is a linear subset of $l$. If $\mathscr{E}=\bigcap_{k=1}^{\infty} \mathscr{E}_{k}$ then $P(\mathscr{E})=1$ and $\mathscr{E}$ is also linear. We define a topology on $\mathscr{E}$ by taking as a base $\mathscr{S}$ translates of sets of the type

$$
\mathscr{U}=\left\{x \in \mathscr{E}:\left(\left(T_{i_{1}}+\cdots+T_{i_{1}}\right) x, x\right)<r\right\}
$$

where $0<r<\infty$ and $\left\{i_{1}, \ldots, i_{j}\right\}$ runs over finite subsets of the integers. That $\mathscr{S}$ is actually a base is easily verified. By Lemma 4.1 it follows that $l_{2}$ is dense in $\mathscr{E}$, and since $\phi(x)$ is uniformly continuous on $l_{2}$ with respect to the topology induced by $\mathscr{S}$ it follows that $\phi(\cdot)$ has the unique uniformly continuous extension $\phi(x)$ $=\lim _{N} \phi\left(P_{N} x\right)$ for $x \in \mathscr{E}$. Furthermore, property (iii) holds for $\phi$ since

for $k=1,2, \ldots$ and $x \in \mathscr{E}$.

$$
\lim _{N}\left(T_{k} Q_{N} x, Q_{N} x\right)=0
$$

Now if $\phi$ is the Fourier transform of a probability measure $\mu$ on $l_{2}$ then $\phi$ is uniformly $\tau$-continuous and hence by Lemma $4.2 \phi$ can be extended to be uniformly continuous on a linear subset $\mathscr{E}$ of $l$ such that $P(\mathscr{E})=1$. However, we have considered another extension of $\phi$ which we called the extended Fourier transform of $\mu$. In this sense $\bar{\phi}$ was defined to be

$$
\tilde{\phi}(x)=\int_{l_{2}} \exp \{i(x, y)\} d \mu(y) \quad(x \in l) .
$$

The next lemma shows that these two definitions agree with probability one. 
LEMMA 4.3. Let $\phi$ be the Fourier transform of a probability measure $\mu$ on $l_{2}$. If $\phi$ and $\mathscr{E}$ are as in Lemma 4.2, and if

$$
\psi(x)=\int_{l_{2}} \exp \{i(x, y)\} d \mu(y) \quad(x \in l),
$$

then $\psi(x)=\tilde{\phi}(x)$ on a linear subset $V$ of $l$ such that $P(V)=1$.

Proof. First of all we see that $\lim _{N}\left(P_{N} x, y\right)=\lim _{N}\left(x, P_{N} y\right)=(x, y)$ almost everywhere $(P \times \mu)$ so by the bounded convergence theorem $\lim _{N} \phi\left(P_{N} x\right)=\psi(x)$ on a linear set $E$ of $l$ such that $P(E)=1$. However, by Lemma $4.2 \phi(x)=\lim _{N} \phi\left(P_{N} x\right)$ for all $x \in \mathscr{E}$ where $P(\mathscr{E})=1$. Thus $\psi(x)=\delta(x)$ for all $x \in V=E \cap \mathscr{E}$ and, clearly, $V$ is a linear subset of $l$ such that $P(V)=1$.

The next lemma is contained in the work of L. Gross [5] but for the sake of completeness we include its proof.

LEMMA 4.4. If $\mu$ and $\nu$ are probability measures on $l_{2}$ then $\mu=\nu$ if and only if their extended Fourier transforms $\tilde{\phi}$ and $\tilde{\psi}$ are equal with probability one on $l$.

Proof. If $\mu=\nu$ it is obvious that $\delta=\tilde{\psi}$ almost everywhere $(P)$ on $l$. The converse goes as follows. If $\mu \neq \nu$ and $\phi$ and $\psi$ are the usual Fourier transforms of $\mu$ and $\nu$, then there exists an $x_{0} \in l_{2}$ such that $\phi\left(x_{0}\right) \neq \psi\left(x_{0}\right)$. Let $h=\phi-\psi$ and assume without loss of generality that $h\left(x_{0}\right)=2 a>0$. Let

$$
I=\left\{x \in l_{2}: h(x)>a\right\} .
$$

Now $h$ is $\tau$-continuous on $l_{2}$ so there exists an $S$-operator $T$ such that if

$$
H=\left\{x \in l_{2}: T\left(x-x_{0}, x-x_{0}\right)<1\right\}
$$

then $H \subseteq I$. If $T(x, x)=\sum_{i} \gamma_{i}\left(x, \alpha_{i}\right)^{2}$ where $\left\{\alpha_{1}, \alpha_{2}, \ldots\right\}$ are orthonormal, $\gamma_{i} \geqq 0$, and $\sum_{i} \gamma_{i}<\infty$ we define

Since

$$
\tilde{H}=\left\{x \in l: \sum_{i} \gamma_{i}\left(x-x_{0}, \alpha_{i}\right)^{2}<1\right\} .
$$

$$
\tilde{h}(x)=\tilde{\phi}(x)-\tilde{\psi}(x)=\lim _{N}\left[\phi\left(P_{N} x\right)-\psi\left(P_{N} x\right)\right]=\lim _{N} h\left(P_{N} x\right)
$$

it follows that $h(x)=\delta(x)-\tilde{\psi}(x) \geqq a>0$ for almost all $x \in \tilde{H}$. This is a contradiction if $P(\tilde{H})>0$. Now

$$
\begin{aligned}
P\{x \in l & \left.: \sum_{i} \gamma_{i}\left(x-x_{0}, \alpha_{i}\right)^{2}<1\right\} \\
& \geqq P\left\{\sum_{i=1}^{N} \gamma_{i}\left(x-x_{0}, \alpha_{i}\right)^{2}<\frac{1}{2} \text { and } \sum_{i=N+1}^{\infty} \gamma_{i}\left(x-x_{0}, \alpha_{i}\right)^{2}<\frac{1}{2}\right\} \\
& =P\left\{\sum_{i=1}^{N} \gamma_{i}\left(x-x_{0}, \alpha_{i}\right)^{2}<\frac{1}{2}\right\} P\left\{\sum_{i=N+1}^{\infty} \gamma_{i}\left(x-x_{0}, \alpha_{i}\right)^{2}<\frac{1}{2}\right\} \\
& \geqq P\left\{\sum_{i=1}^{N} \gamma_{i}\left(x-x_{0}, \alpha_{i}\right)^{2}<\frac{1}{2}\right\}\left[1-2 \sum_{i=N+1}^{\infty} \gamma_{i}\right]
\end{aligned}
$$


where the equality holds because $\left\{\left(x-x_{0}, \alpha_{i}\right)\right\}$ is a sequence of independent Gaussian random variables and the last inequality follows by Čebyšev's inequality and the fact that $E\left[\left(x-x_{0}, \alpha_{i}\right)^{2}\right]=1$. Hence for $N$ such that $\left[1-2 \sum_{i=N+1}^{\infty} \gamma_{i}\right]$ is positive, we have the last quantity as a product of two positive numbers and we see that $P(\tilde{H})>0$. This is a contradiction so $\mu=\nu$ as was to be proved.

We now prove Gross' result for $l_{2}$.

THEOREM 4.1. If $\left\{\mu_{k}\right\}$ is a sequence of probability measures on $l_{2}$ with Fourier transforms $\left\{\phi_{k}\right\}$, then $\left\{\mu_{k}\right\}$ converges weakly to a measure $\mu$ if and only if $\left\{\tilde{\phi}_{k}\right\}$ converges in probability to $\bar{\phi}$ for some $\tau$-continuous $\phi$ on $l_{2}$ such that $\phi(0)=1$.

Proof. If $\left\{\mu_{k}\right\}$ converges weakly to $\mu$ then $\left\{\tilde{\phi}_{k}\right\}$ converges in probability to $\tilde{\phi}$, the extended Fourier transform of $\mu$, by the first part of Theorem 3.1. Furthermore, $\phi(0)=1$ and $\phi$ is $\tau$-continuous on $l_{2}$. Conversely, we assume $\left\{\phi_{k}\right\}$ converges to $\{\tilde{\phi}\}$ in probability, where $\phi(0)=1$ and $\phi$ is $\tau$-continuous on $l_{2}$ (here, of course, $\tilde{\phi}$ is the continuous extension of $\phi$ as given in Lemma 4.2). Let $J_{N, \gamma}\left(\mu_{k}\right)$ be as in Lemma 3.3 with $p=2$. We now verify that

$$
\lim _{N \rightarrow \infty ; \gamma \downarrow 0} \sup _{k} J_{N, \gamma}\left(\mu_{k}\right)=0
$$

under the present hypothesis. In fact, the proof proceeds exactly as in Lemma 3.3 except that

$$
\lim _{N \rightarrow \infty ; \gamma \downarrow 0} \int_{l}\left[1-\delta\left(\gamma P_{N} x+Q_{N} x\right)\right] d P(x)=0
$$

because $\bar{\phi}$ is a continuous function on a linear subset of $l$ of probability one and by Lemma 4.2

$$
\lim _{N \rightarrow \infty ; \gamma \downarrow 0} \tilde{\phi}\left(\gamma P_{N} x+Q_{N} x\right)=\tilde{\phi}(0)=\phi(0)=1 .
$$

Thus by Lemma $3.2\left\{\mu_{k}\right\}$ is conditionally compact, and proceeding as in Theorem 3.1 (along with Lemma 4.4) we see $\left\{\mu_{k}\right\}$ converges weakly to a measure $\mu$ with extended Fourier transform $\tilde{\phi}$.

5. The continuity theorem on $l_{p}, 2 \leqq p<\infty$, involves the concept of a $\lambda$-family of measures as defined in $\S 2$.

If $\lambda$ is in the positive cone of $l_{p / 2}^{*}$ we will denote by $P_{\lambda}$ the probability measure on $l$ which has the coordinate functions as independent Gaussian random variables with mean zero and $E\left(\mathscr{P}_{k} x\right)^{2}=\lambda_{k}$. For $p=2$ we have $l_{p / 2}^{*}=l_{\infty}$ and we can choose $\lambda=(1,1, \ldots)$ so $P_{\lambda}$ then denotes the canonical Gaussian distribution on $l$ used in $\S \S 3$ and 4 and in [3], [4], [5].

If $\mu$ is a probability measure on $l_{p}$ with Fourier transform $\phi$ defined on $l_{p}^{*}$, then the $P_{\lambda}$-extended Fourier transform is defined on $l$ as follows:

$$
\tilde{\phi}(x)=\int_{l_{p}} \exp \{i(x, y)\} d \mu(y) \quad(x \in l) .
$$


Using the ideas of Lemma 3.1 and the fact that $\lambda \in l_{p / 2}^{*}$ it is easy to see that $\phi(x)$ is a measurable function on $l$. When dealing with a version of $\phi$ we will always assume that it agrees with $\phi$ on $l_{p}^{*}$.

THEOREM 5.1. Let $\left\{\mu_{k}\right\}$ be a sequence of probability measures on $l_{p}, 2 \leqq p<\infty$, with Fourier transforms $\left\{\phi_{k}\right\}$. Then $\left\{\mu_{k}\right\}$ converges weakly to a measure $\mu$ with Fourier transform $\phi$ if and only if $\left\{\mu_{k}\right\}$ is a $\lambda$-family for some $\lambda$ in the positive cone of $l_{p / 2}^{*}$, $\left\{\bar{\phi}_{k}\right\}$ converges in $P_{\lambda}$-measure to $\phi$, and $\left\{\phi_{k}\right\}$ converges to $\phi$ on $l_{p}^{*}$.

Proof. Suppose $\left\{\mu_{k}\right\}$ converges weakly to $\mu$. Then $\left\{\mu_{k}\right\}$ is conditionally compact and by Lemma 2.2 it is a $\lambda$-family for any $\lambda$ in the positive cone of $l_{p / 2}^{*}$. Repeating the argument given in the proof of Theorem 3.1 it follows that $\left\{\phi_{k}\right\}$ converges in mean-square to $\dot{\phi}$ and hence $\left\{\tilde{\phi}_{k}\right\}$ converges in $P_{\lambda}$-probability to $\dot{\phi}$. That $\left\{\phi_{k}\right\}$ converges to $\phi$ on $l_{p}^{*}$ is easy.

Now assume $\left\{\tilde{\phi}_{k}\right\}$ converges in $P_{\lambda}$-probability to $\tilde{\phi}$. If

$$
\begin{aligned}
H_{N, \gamma}\left(\mu_{k}\right) & =\int_{l_{p}}\left[1-\exp \left\{-\frac{1}{2}\left[\sum_{j=1}^{N} \lambda_{j} \gamma^{2} y_{j}^{2}+\sum_{j=N+1}^{\infty} \lambda_{j} y_{j}^{2}\right]\right\}\right] d \mu_{k}(y) \\
& =\int_{l}\left[1-\tilde{\phi}_{k}\left(\gamma P_{N} x+Q_{N} x\right)\right] d P_{\lambda}(x)
\end{aligned}
$$

then

$$
\begin{aligned}
\lim _{k \rightarrow \infty} H_{N, r}\left(\mu_{k}\right) & =\int_{l}\left[1-\delta\left(\gamma P_{N} x+Q_{N} x\right)\right] d P_{\lambda}(x) \\
& =\int_{l_{p}}\left[1-\exp \left\{-\frac{1}{2}\left[\sum_{j=1}^{N} \lambda_{j} \gamma^{2} y_{j}^{2}+\sum_{j=N+1}^{\infty} \lambda_{j} y_{j}^{2}\right]\right\}\right] d \mu(y)
\end{aligned}
$$

and hence

$$
\lim _{N \rightarrow \infty: \gamma \downarrow 0} \sup _{k} H_{N, \gamma}\left(\mu_{k}\right)=0 .
$$

Thus for $\varepsilon, \delta>0$ there exists $\gamma_{0}>0, N_{0}$ such that $\gamma \leqq \gamma_{0}$ and $N \geqq N_{0}$ implies

$$
\mu_{k}\left\{x \in l_{p}: \gamma^{2} \sum_{j=1}^{N} \lambda_{j} x_{j}^{2}+\sum_{j=N+1}^{\infty} \lambda_{j} x_{j}^{2}<\delta\right\} \geqq 1-\varepsilon
$$

for $k=1,2, \ldots$ (see Lemma 3.2 for this type of argument). Since $\left\{\mu_{k}\right\}$ is a $\lambda$-family we thus have for $\gamma$ sufficiently small ( $\gamma$ may depend on $N$ ) that

$$
\mu_{k}\left\{x \in l_{p}:\left\|\gamma P_{N} x+Q_{N} x\right\|^{p}<\delta\right\} \geqq 1-\left(2 \varepsilon+\varepsilon_{N}\right)
$$

where $\lim _{N} \varepsilon_{N}=0$. Then, as in Lemma 3.2, we see that $\left\{\mu_{k}\right\}$ is conditionally compact and since $\left\{\phi_{k}\right\}$ converges to $\phi$ on $l_{p}^{*}$. We have $\left\{\mu_{k}\right\}$ converging weakly to $\mu$.

As a final remark we mention that using the above techniques it is possible to prove a central limit theorem for independent indentically distributed random variables in $l_{p}, 0<p<\infty$. In the case $1 \leqq p \leqq 2$ certain results are given in [7] and [11]. 


\section{REFERENCES}

1. R. H. Cameron and R. E. Graves, Additive functionals on a space of continuous functions. I, Trans. Amer. Math. Soc. 70 (1951), 160-176. MR 12, 718.

2. J. Feldman, $A$ short proof of the Lévy continuity theorem in Hilbert space, Israel J. Math. 3 (1965), 99-103. MR 33 \#766.

3. L. Gross, Integration and non-linear transformations in Hilbert space, Trans. Amer. Math. Soc. 94 (1960), 404-440. MR 22 \#2883.

4. - Measurable functions on Hilbert space, Trans. Amer. Math. Soc. 105 (1962), 372-390. MR 26 \#5121.

5. - Harmonic analysis on Hilbert space, Mem. Amer. Math. Soc. No. 46 (1963). MR 28 \#4304.

6. M. Loéve, Probability theory, 3rd ed., Van Nostrand, Princeton, N. J., 1963. MR 23 \#A670.

7. Ju. V. Prohorov, Convergence of random processes and limit theorems in probability theory, Teor. Verojatnost. i Primenen. 1 (1956), 177-238= Theor. Probability Appl. 1 (1956), 157-214. MR 18, 943.

8. Ju. V. Prohorov and V. Sazonov, Some results related to Bochner's theorem, Teor. Verojatnost. i Primenen. 6 (1961), 87-93 = Theor. Probability Appl. 6 (1961), 82-86. MR 26 \#6729.

9. V. Sazonov, On characteristic functionals, Teor. Verojatnost. i Primenen. 3 (1958), 201-205 = Theor. Probability Appl. 3 (1958), 201-205. MR 20 \#4882.

10. N. N. Vakhania, Sur une propriété des répartitions normales de probabilités dans les espaces $l_{p}(1 \leqq p<\infty)$ et $H$, C. R. Acad. Sci. Paris 260 (1965), 1334-1336. MR 30 \#4282.

11. - Sur les répartitions de probabilitiés dans les espaces de probabilité dans les espaces de suites numériques, C. R. Acad. Sci. Paris 260 (1965), 1560-1562. MR 30 \#4283.

UNIVERSITY OF WISCONSIN,

Madison, WisCONSIN 53706

Mathematics Research Center, United States Army, MAdison, Wisconsin 53706 